\title{
Research on the Application of Evidence-based Thought in the Prevention and Response to Non-traditional Security Threats in Xinjiang
}

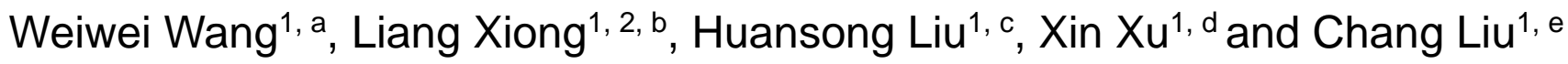 \\ ${ }^{1}$ Military Economic Academy, Wuhan 430035, China; \\ ${ }^{2}$ Social Science Academy of Xinjiang, Urumqi 830001, China. \\ a35582368@qq.com, bmilitaryxl@163.com, cliuhuansong@x263.net, dxuxin@163.com, \\ eliuchang7j@vip.qq.com
}

\begin{abstract}
The application of evidence-based thought in the prevention and response of the non-traditional security threats in Xinjiang aims to ensure making decisions based on the best accessible research evidence, and introduce the method of transparently accessing and evaluating the evidence into the whole decision making process, the substance of which is to concern facts and analyze situation. According to the general requirements of evidence-based thought, taking advantage of the Agent Technology with its powerful openness, flexibleness, adaptability and cooperation, to construct the decision making system of the prevention and response of the non-traditional security threats in Xinjiang is able to highly meet the requirement of high practicality, high risks and strong dynamics of the prevention and response of the non-traditional security threats in Xinjiang, and will prove to provide more effective and reliable new way of thinking for the prevention and response of the non-traditional security threats in Xinjiang, with its objectivity, simplicity and superiority of evidence-based analyses, process and conclusion.
\end{abstract}

Keyword: Evidence-based; non-traditional security; Xinxiang; threat defense; threat response.

\section{Introduction}

At present, our country is facing unprecedented non-traditional security challenges, and the threat to national security and development worsen the situation [1]. As an important part of national security strategy, Xinjiang's non-traditional security threats have in common with other provinces in China [2]. At the same time, because it is located in the northwest frontier, multi-ethnic-populated, multi-religious intersected, and multi-culture collided, its non-traditional security threats also has a strong personality, is more complicated, more intertwined and far-reaching [3-7].

To prevent and deal with non-traditional security threats in xinjiang is of much practicality. It has to conduct the operation closely according to our national conditions and the situation in xinjiang [8]. From the practical effect of guarding against and dealing wtih the non-traditional security threats in xinjiang in recent years, the traditional thinking way of its prevention and response has made great achievement [9]. In the meantime, we should also clearly realize that due to the practice earlier than theory in the awareness of non-traditional security threats in xinjiang and its further study is only in recent years, there are some limitations and defects both in the thinking way of its prevention and response and in its strategy. Therefore, it will have extremely important theoretical significance and practical value to promote the innovation of the thinking way in xinjiang's non-traditional security threats prevention and response and use it to guide practice. From the angle of view of the Evidence-based Decisions, this article try to explore the new ideas in the prevention and response of the non-traditional threats to security in Xinjiang [10-15].

\section{Evidence-based Decisions: Inspired by Evidence-based Medicine}

The traditional medical treatment activity is a kind of experience and experiment. A doctors cures 
according to his own clinical experience and reference from the experience of other doctors at the same time; makes interrogation and examination using the textbook design of diagnosis and treatment and under the condition of insufficient or inconclusive evidence, supplemented by laboratory test; make a judgment according to already controlled evidence; uses drugs tentatively and at the same time observes the condition change during the period of treatment and the different reactions after medicine; and then obtains new evidence for the treatment being effective and feasible; yet again decides whether or not to continue to use associative drugs as well as how to adjust the treatment program. And the evidence-based medicine (ebm) developed in the 1970s provides a new train of thought for disease diagnosis and treatment. Evidence-based medicine refers to "deliberately, accurately and intelligently apply the best research evidence we can get to determine the patient treatment measures".It emphasizes the organic combination of the three elementsthe: clinical evidence, doctor's individual experience and patient's actual situation and willingness. In other words, Evidence-based medicine is based on the objective and follow the objective and keep dynamic diagnosis and treatment of disease, it has given expression to the change from personal experience to the evidence, and a relationship of mutual promotion between theory and practice.

In some ways, the prevention and response of the non-traditional threats to security in Xinjiang is similar with medicine, such as highly practical, uncertainty, as well as the dynamic characteristics of the development and so on. The traditional treatment method seems similar to the prevention and response of the current non-traditional threats to security in Xinjiang. It developes in the mode of "experience + experiment", whether the prevention activities of non-traditional threats to security such as preplan establishing or the choice of corresponding positive measures. The traditional way of prevention and response does exist some shortcomings, for instance, experience can reject unknown emerge technology, fresh methods and new ideas; The wrong experience and practice is formed due to the complexity of the causal relationship; And it proved effective through theoretical derivation, but its actual effect is not likely conform to the experiment conclusion or theoretical demonstration. It would be necessary as well as feasibl that we redesign the thinking way of the prevention and response of the Xinjiang non-traditional threats to security borrowing ideas from the achievement of evidence-based medicine.

\section{Basic Procedure}

Under normal circumstances, the evidence-based decision of the prevention and response of the Xinjiang non-traditional threats to security can be divided into five steps, namely, the problems are put forward $\rightarrow$ the evidences are collected( retrieved) $\rightarrow$ the evidences are evaluated and analysed $\rightarrow$ using evidence to guide practice $\rightarrow$ and evaluating the effectiveness. Unlike formerly decision method, evidence-based process is a procedure of continuous cycle, the conclusion of the previous evidence-based decisions will become the new data and evidence to update the proof database, and become the new evidence for the next decision.

Asking question, that is, identify the principal problem to make decision. Normally, the suggested problem has two main parts: The first is a question of practicality, such as the specific work flow, profession skill, and the problems in the daily operation process to prevent and deal with non-traditional security threats in Xinjiang. For example, Xinjiang in springtime is vulnerable to the threat of snowmelt flood disaster, to prevent and cope with the threat requires finding the preventing way, reacting process, and coping strategies to the threat by adoption of evidence-based method. The second is a question of involving for further research, such as the innovation of the concept, the model and the system of the prevention and response of the Xinjiang non-traditional threats to security. Also as an example, with the dynamic variation of the non-traditional threats to security faced with Xinjiang, every once in a while we should evaluate the existing threat condition, this is where the problem is presented by evidence-based way, such as "predicting the key point to the Xinjiang non-traditional 
threats to security in year to come", "establishing the macroscopic strategy answering to Xinjiang non-traditional threats to security for the next five years" and more. It is worth noting that the suggested questions must be clearly specified.

Collecting (retrieving) evidence, that is, look for the evidence supports as evidence-based evidence needed through a variety of effective way. This is the premise of evidence-based thinking in the prevention and response of the Xinjiang non-traditional threats to security. The evidence-based decision making in the prevention and response of the Xinjiang non-traditional threats to security has the evidence of a wide range of sources, such as personal experience, expert knowledge and iterature etc. All the evidence related to the "problem" need to be collected, and the evidence is more complete the decision conclusion will be more scientific. Take the prevention and response to the threat of terrorism in xinjiang as an example, the evidence need to be collected mainly relates to hostile situation, our situation and natural conditions, etc., the content of evidence mainly includes laws and regulations, national policy, counter-terrorism experience, theoretical achievements, action practice, and drill data. These evidence comes from everything from all kind of database to a wide array of information and expertise. It is important to note that to clarify the relationship between evidence and information, the evidence is information, but the information is not necessarily evidence -- only through scientific processing information constitutes the evidence.

Evaluating and analysing evidence, that is, rank all of evidence collected according to argumentation intensity, and conduct validity check with scientific method. This is the foundation of evidence-based thinking in the prevention and response of the Xinjiang non-traditional threats to security. With different sources of evidence and widely forms, the proven strength of different evidence to the facts is not the same. Also take the prevention and response of the terrorism threat as an example, from the point of evidence form, there are mainly environment evidence, oral evidence, written evidence, physical evidence, electronic evidence, etc., but in general, written evidence is more reliable than oral evidence and directly obtained evidence is more effective than indirect obtained evidence. Therefore, we must reasonably evaluate the evidence we have obtained and confirm its reliability and rationality in search of the best effective and most reasonable evidence to support the activity practice of the prevention and response of xinjiang non-traditional security threats.

Guidind practice by evidence means supporting the decision making of the prevention and response of xinjiang non-traditional security threats with the evaluated evidence, and directing the specific work of prevention and response with the results of decision-making. This is at the heart of evidence-based thinking in the prevention and response of the Xinjiang non-traditional threats to security. In fact, the evidence collection and evaluation is the prelude of the decision-making based on the evidence-based prevention and response of Xinjiang non-traditional security threats, applying the best and most reasonable evidence that have already obtained and using it guide practice are all that matters. This is a complicated process, and there is the need of specialized information system to complete.

Evaluation results means using the practice to verify the conclusions based on evidence-based decisions. This is the end of an evidence-based decisions, and at the same time it will be the beginning of the next evidence-based decisions -- the conclusion of evidence-based decision-making after evaluation will be updated into the database, and the decision conclusion will become a new evidence to provide a new basis for the next evidence-based decisions.

The evidence-based decision-making process of the prevention and response of Xinjiang non-traditional threats to security, as shown in fig. 1. From the key elements of evidence-based management -- evidence, the evidence-based decision-making process of the prevention and response of Xinjiang non-traditional security threats, has always developed closely around "evidence", in which all the data that we have collected will first be summarized to serve as information beneficial to solve the problem, all kinds of information producing evidence through processing, and finally guiding practice and drawing a conclusion by collecting, retrieving, evaluating, analysing and utilising, and at the same time providing the new evidence; If from the angle of thinking, the evidence-based decision 
process of the prevention and response of Xinjiang non-traditional threats to security, here again has been developed centering on the procedure of "asking questions - analysising problem - solving problem", which is consonant with the objective laws the people think.

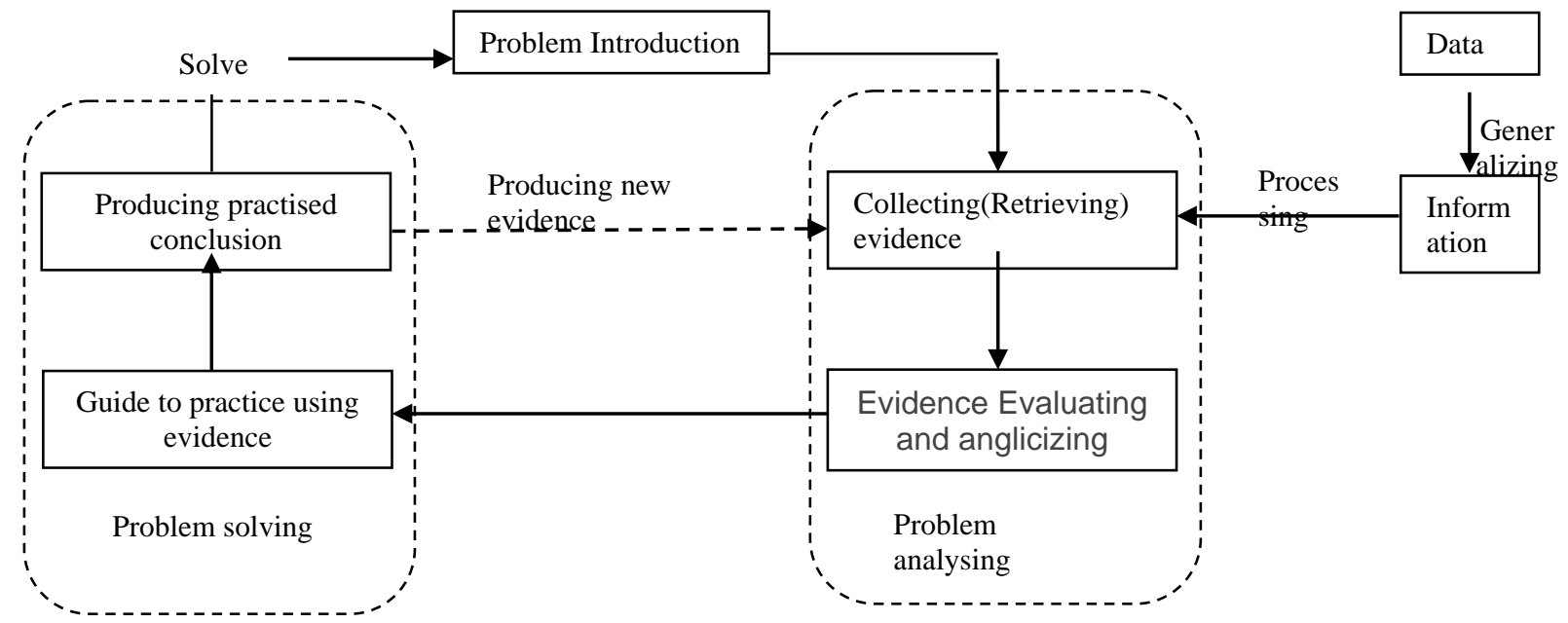

Fig.1 The evidence-based decision-making process of the prevention and response of Xinjiang non-traditional threats to security

\section{Model Building}

The prevention and response of Xinjiang non-traditional security threats has always been born companion with decision-making, the advantages and disadvantages of the decision-making process is largely affect the moving ahead of the prevention and response work. The traditional decision support method when people make decisions has played a large role, but also there are some defects, such as incapable active to summarize the existing data information, unable to make effective evaluation to existing evidence, and incapable offering reasonable suggestions according to the changes in the environment; At the same time, it also can not make full use of the resources according to the environmental variation; It often adopts centralized controlling means, which limits the intellectual instruction of the components of the system in the distributed state to decision-making system. And these are based on the evidence-based functione vital for the prevention and response of Xinjiang non-traditional threats to security. Current popular and more advanced Agent modeling method can handle this kind of problem very well. Therefore, in this article I will build the decision-making system of the prevention and response of Xinjiang non-traditional threats to security, based on Agent technology.

The evidence-based decision-making model for the prevention and response of Xinjiang non-traditional threats to security direct map each member participating in the decision-making process in the field world for each Agent and encapsulate this members' resource, knowledge, target and ability as the Agent own properties, thus achieve the purpose of simulating or acting the actual component. On the basis of normalized description process of the prevention and response of xinjiang non-traditional threats to security, Agent evidence-based decision-making model using Agent technology conduct analysis and design, and compile the corresponding Agent for each system and department involved in the working process to make each Agent with knowledge, resource and inferential capability of corresponding departments, its purpose is to make each Agent to provide the corresponding department and system with decision support in such areas as task execution way and method. The overall framework of evidence-based decision making system in the prevention and response of Xinjiang non-traditional threats to security is shown in Fig. 2. 


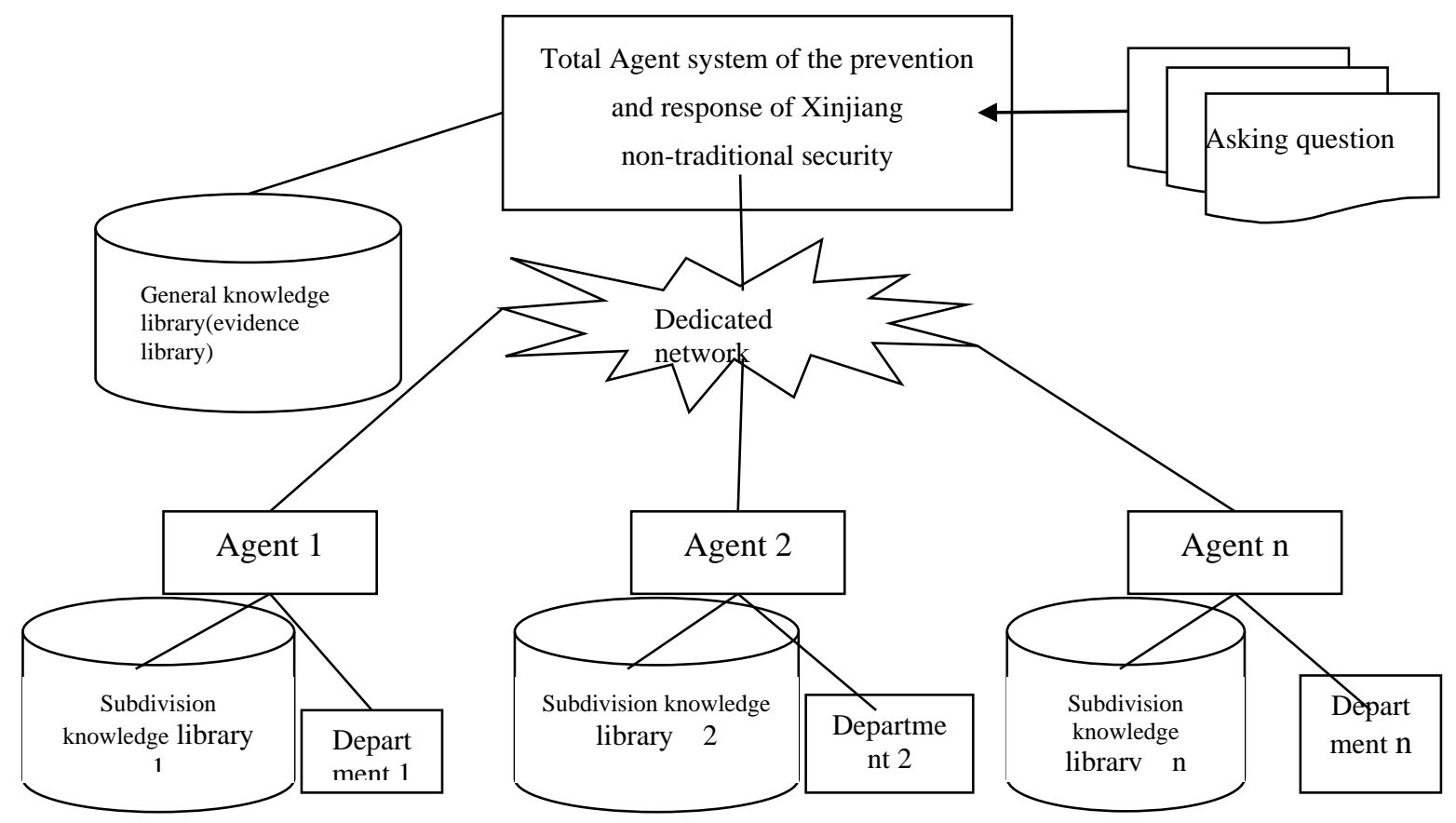

Fig.2 The overall framework of evidence-based decision-making system to prevent and deal with non-traditional security threats in Xinjiang

For internal function modules in each Agent, normally, mostly including sense, communication, reasoning, execution module, internal state library and knowledge base, its basic structure is shown in Fig. 3.

Such system architecture is provided with the following define feature: First, Autonomy. That is to say Agent can be run up under the condition of nobody or no other Agent's direct intervention, and have some sort of control ability for its own behavior and internal states. This feature allows the user to focus on a high level of targets, while let the Agent take charge of completing the detail procedure of these targets, the Agent can sense according to its own knowledge, state and the perception of the external environment, and bring with the decision what to do, does not require direct intervention of the outside environment. Second, Responsivity. That is to say Agent can observe its environment, such as the physical world, user using the graphical interface, other Agent, network and the combination of these factors, and react in a certain period of time so as to change the environment. Third, sociability. That is to say Agent can interact with other Agent to solve the problem of itself or other Agent. Fourth, Pre-activeness. That is to say Agent can not only respond to the environment, but also can initiate to show (plan or start) the targeted action: target oriented, initiating action, rather than simply environmental adaptation. 


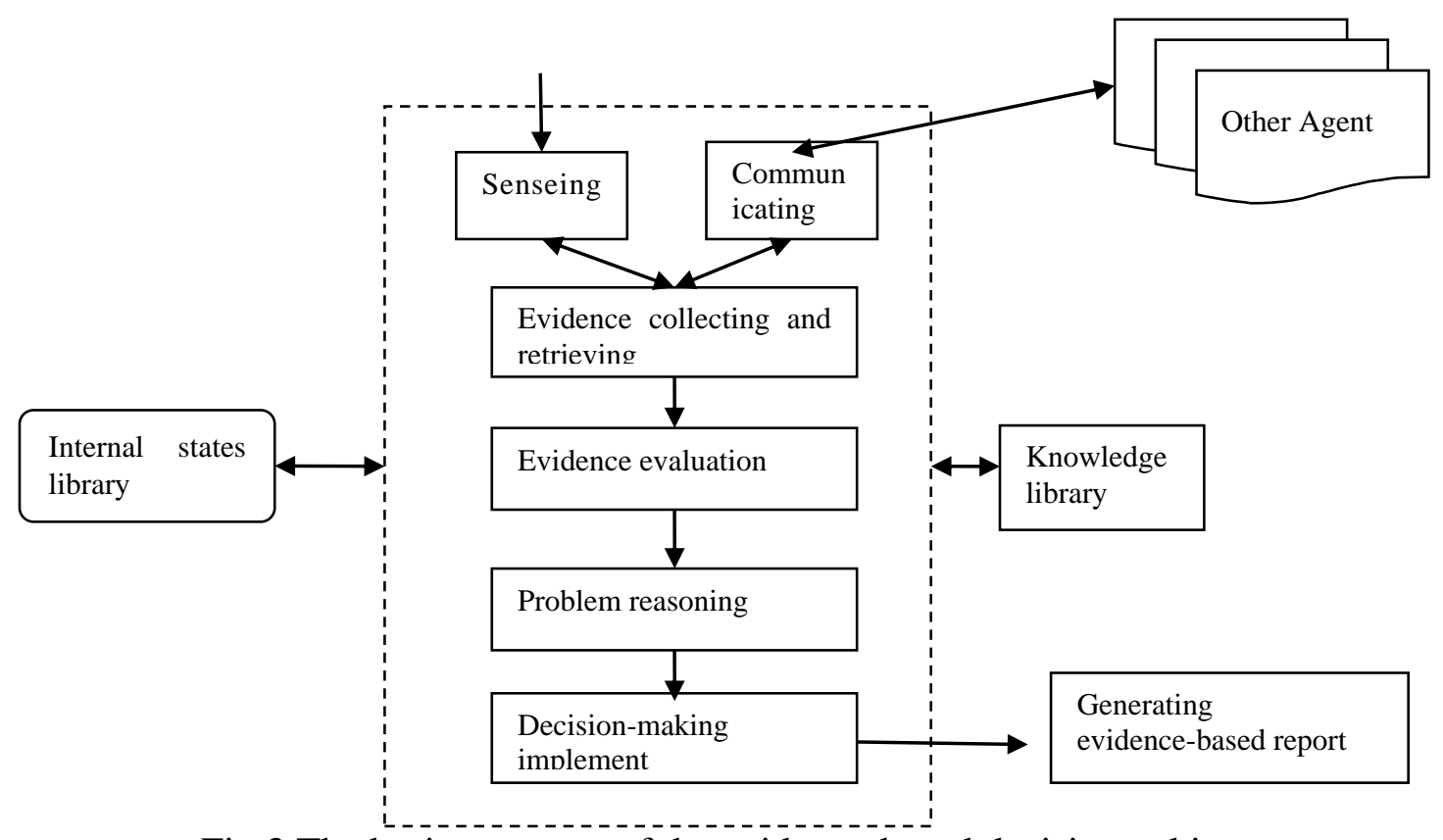

Fig.3 The basic structure of the evidence-based decisionmaking system

\section{Conclusion}

The process of the prevention and response in Xinjiang non-traditional threats to security is, in fact, the formation process of a series of decision-making. Due to the reasons such as the objective differences faced with the threat, the reality of limited rational decision making, the gap between theory research and practice and the actual deviation of empirical decision-making, the prevention and response of Xinjiang non-traditional threats to security under the traditional thinking way has objectively some limitations. The application of evidence-based theory in the prevention and response of the non-traditional security threats in Xinjiang aims to ensure making decisions based on the best accessible research evidence, and introduces the method of systematiclt and transparently accessing and evaluating the evidence into the whole decision making process, the substance of which is to concern facts and analyze situation. In accordance with the general requirements of evidence-based thought and taking advantage of the Agent technology predominance suh as its powerful openness, flexibility, adaptability and collaborativeness, building the decision making system of the prevention and response of Xinjiang non-traditional threats to security can effectively overcome the limitations of the traditional way of thinking and adapt to the requirements of high practicality, high venture nature and strong dynamics in the prevention and response of Xinjiang non-traditional threats to security. With the objectivity of evidence-based analysis, the simplicity of its process and the superiority of its conclusion, these predominance will provide the prevention and response of Xinjiang non-traditional threats to security with more effective and reliable new ideas.

\section{References}

[1] Weng Hua, From evidence-based medicine to evidence-based management [J]. Managerialist, 2011(10).

[2] Sun Lin, Tang $\mathrm{Yu}$ and Xie lei et. al., The hospital logistics research and application of evidence-based management [J]. China Hospital, 2013 (11).

[3] Jiang Yong, A study on detection evidence-based decision-making in risk society [J]. Journal of the Chinese People's Public Security University, 2013 (5). 
[4] Zhou Zhiren and Li Le, Evidence-based decisions: international practice, theory origin and academic position [J]. Journal of Chinese Administrative Management, 2013 (12).

[5] Zhang Lulu, The research and developments of health evidence-based decision-making [J]. Journal of Second Military Medical University, 2012 (8).

[6] Wei Dang, Wang Qi and Sun Li et. al., From evidence-based decision to evidence-informed decision [J]. Journal of Gansu Science and Technology, 2013 (9).

[7] (US) Ross C. Bronson, Elizabeth A. Beek and Trie L. Littel et. al., The Evidence-Based Public Health [M]. Beijing: Beijing Union Medical University Press, 2012.12.

[8] An Xiaoping, Xi Guiping and Li Xinming, A Tentative Study on the particularity of non-traditional security threats in Xinjiang [J]. Journal of Tarim University, 2010 (3).

[9] Liu Yuezhen, An analysis of Non-traditional security challenges and countermeasures in Xinjiang [J]. The Border Economy and Culture, 2009 (8).

[10] Zhu Qin, Harmonious Governance in the Frontier Minority Area: in the Framework of Emergency Management [M]. Kunming: Yunnan People's Publishing House, October 2009.

[11] Lu Pangliang and Chen Guoshun, Research on smart remote monitoring system of command and control equipment based on multi-Agent [J]. Computer \& Digital Engineering, November 2014.

[12] Guo Chao and Xiongwei, Unit confronting modeling and simulation based on multi-Agent system [J]. Command Control \& Simulation, 2015 (4).

[13] Wang Pingli and Wu Zheng, Emergency command platform of military operations other than war based on multi-Agent technology [J]. Ordnance Industry Automation, 2014 (2).

[14] Liu Dan, Cao Jiantong and Chen Jianming, Applications based on Agent modeling: research review [J]. Journal of Technology Economy, 2014 (11).

[15] Song Huawen, Wang Pinghui and Wang Yu, Research on intelligent decision support sysytem of ammunition supply control based on Agent [J]. Journal of Academy of Equipment Command \& Technologyy, 2001 (4). 